\title{
Editorial \\ "The Compromised Fetal and Neonatal Lives and their Possible Long-term Consequences"
}

This special issue concerns several topics about the possible long-term consequences when a given life, during his fetal and neonatal periods, is compromised. I remember, when I was resident in neonatal intensive care (that was in the seventies, hence forty years ago), how fascinated I had been by the following sentence: "Time present and time past are both perhaps present in time future, and time future contained in time past" -Burnt Norton, Thomas Staerns Eliot. I sincerely couldn't, at that time, realize the global meaning of these words

Research in perinatal medicine has been induced by great pioneers such as among many examples: Sir Geoffrey Dawes in Oxford, Mary Ellen Avery in Boston and afterwards by many other around the world. It allowed to discover many aspects that will be described in The Introduction. ions:

It is my great pleasure to present to our readers the work about the following aspects from my collaborators and compan-

$>$ Concerning the consequences of intra-uterine growth retardation: from the group of Olivier Baud [1].

$>$ Concerning renal and cardiovascular aspects: from the group of Umberto Simeoni [2].

$>$ Concerning the potential of stem cells in fragile premature, pulmonary aspects: from the group of Bernard Thebaud [3].

I wish to dedicate this manuscript to Wivinne Marion a marvellous mother and neonatologist who died unfortunately at 42 years.

\section{REFERENCES}

[1] M. Colella, A. Frérot, A.R.B. Novais and O. Baud. Neonatal and Long-Term Consequences of Fetal Growth Restriction. Curr Pediatr Rev 2018; 14(4): 212-18.

[2] H. Chehade, U. Simeoni, J-P. Guignard and F. Boubred. Preterm Birth: Long Term Cardiovascular and Renal Consequences. Curr Pediatr Rev 2018; 14(4): 219-26

[3] M.T. Ee and B. Thébaud. The Therapeutic Potential of Stem Cells for Bronchopulmonary Dysplasia: "It's About Time" or "Not so Fast" ?. Curr Pediatr $\operatorname{Rev} 2018$; 14(4): 227-38.

Oreste Battisti

(Guest Editor)

Emeritus Academic Professor Oreste Battisti, University of Liège (Belgium) And Catholic University of Bukavu Democratic Republic of the Congo

E-mails: Oreste.battisti@ulg.ac.be; oreste.battisti@uliege.be 